\title{
Structure in cohesive powders studied with spin-echo small angle neutron scattering
}

\author{
R. Andersson - W. G. Bouwman - S. Luding • \\ I. M. de Schepper
}

Received: 21 March 2007 / Published online: 26 June 2008

(C) The Author(s) 2008

\begin{abstract}
Extracting structure and ordering information from the bulk of granular materials is a challenging task. Here we present Spin-Echo Small Angle Neutron Scattering Measurements in combination with computer simulations on a fine powder of silica, before and after uniaxial compression. The cohesive powder packing is modeled by using molecular dynamics simulations and the structure, in terms of the density-density correlation function, is calculated from the simulation and compared with experiment. In the dense case, both quantitative and qualitative agreement between measurement and simulations is observed, thus creating the desired link between experiment and computer simulation. Further simulations with appropriate attractive potentials and adequate preparation procedures are needed in order to capture the very loose-packed cohesive powders.
\end{abstract}

Keywords SESANS - Powder - Cohesion - Structure · Density correlation $\cdot$ Molecular dynamics

R. Andersson $(\varangle)$ · W. G. Bouwman · I. M. de Schepper

Radiation, Radionuclides and Reactors,

Delft University of Technology, Mekelweg 15,

2629JB Delft, The Netherlands

e-mail: r.a.andersson@tnw.tudelft.nl; r.a.andersson@tudelft.nl

S. Luding

Nanostructured Materials, DCT,

Delft University of Technology,

Julianalaan 136, 2628 BL Delft, The Netherlands

S. Luding

Multi Scale Mechanics, TS, CTW

Department of Mechanical Engineering,

University of Twente, P.O. Box 217,

7500 AE Enschede, The Netherlands

e-mail: s.luding@utwente.nl

\section{Introduction}

The macroscopic properties of a granular material are given by the mutual arrangement of the grains and its geometry, i.e., its structure. However, structure in granular materials is usually discussed in the framework of random spherepackings [1], the classification and underlying theory describing the packings of grains are yet to be found, and more experiments are needed. The structure in granular materials is not only of fundamental importance, but also of great practical relevance in industry related applications. Powders with cohesive properties are widely handled throughout industry, either as a raw material, by-products, or as the desired end product. Considering fine powders (micro meter sized), inter-particle forces such as van der Waals, electrostatic and capillary forces influences the structure of the packing [2], leading to clustering [3], channelling and tunnelling effects in fluidised beds, and oscillating mass flows in general [4].

Various experimental methods to extract structural information in granular materials do exist. Magnetic Resonance Imaging has been proven successful in determining the structure of non-cohesive granular packings [5], and even dynamic measurements have been carried out [6]. Confocal microscopy has been applied to construct 3D images of granular materials immersed in a refraction-index matched liquid [7, 8]. X-ray computed microtomography followed by a tomographic reconstruction is a powerful 3D method that maps out the density in dry packings of, usually, model granular materials [9-11].

Cohesive powders are complex systems with a typical grain size not readily accessible with the techniques mentioned above. The use of conventional wave diffraction techniques such as small-angle neutron scattering (SANS) are commonly applied for the study of mesoscopic bulk structures (colloids, polymers, macromolecules etc.) [12]. The 
resolution of a conventional SANS instrument does not allow for the study of powders at the grain level, and one is usually limited to a few $100 \mathrm{~nm}$. At the Delft University of Technology a novel SANS technique has been developed [13, 14]. Spin-Echo small angle neutron scattering (SESANS) enables measurements over three orders of magnitude, ranging from $30 \mathrm{~nm}-20 \mu \mathrm{m}$ in length [15], making this technique applicable for probing the bulk-structure in fine powders $[16,17]$.

Here we report measurements on a fine cohesive powder of silica before and after uniaxial compression. The measurement shows how larger structures disappear upon compression. In the loose-packed state we observe correlations extending far beyond the average size of a single grain. Molecular dynamics simulations were carried out in order to model the measurement. We find, in the dense case, good quantitative and qualitative agreement with the measurement. A loose packed sample is only in qualitative agreement, showing that more computational work is needed in order to capture the structures formed with low packing fractions.

\section{Experiment, the sample and measured quantities}

\subsection{SESANS: Measured quantities}

Spin-Echo SESANS, is based on the Larmor precession of neutrons in tilted magnetic fields $[18,19]$. In SESANS, the polarisation of a neutron beam is measured, after transmission through a sample, as a function of the so-called spinecho length, $z$ ( $30 \mathrm{~nm}<z<20 \mu \mathrm{m})$. The spin-echo length is the length at which density correlations are measured. The measured polarisation is directly related to the projection of the sample density-density correlation function along the neutron beam path. In particular, for a two-phase system we have, for the transmission of polarisation, normalised with experimental effects:

$$
P(z)=e^{t \lambda^{2} \phi(1-\phi) \Delta \rho_{0}^{2} \xi(G(z)-1)},
$$

where $G(z)$ is the normalised SESANS correlation function, representing the projection of the density-density correlation function of the sample (see Fig. 1). $\phi$ is the volumefraction, $t$ the sample thickness, $\lambda$ is the neutron wavelength and finally we have $\Delta \rho_{0}$ being the neutron scattering length density (SLD) [20] difference between the two phases. We write the projection of the density-density correlation function, $\gamma(r)$, as:

$$
G(z)=\frac{2}{\xi} \int_{z}^{\infty} \frac{r \gamma(r)}{\sqrt{r^{2}-z^{2}}} d r,
$$

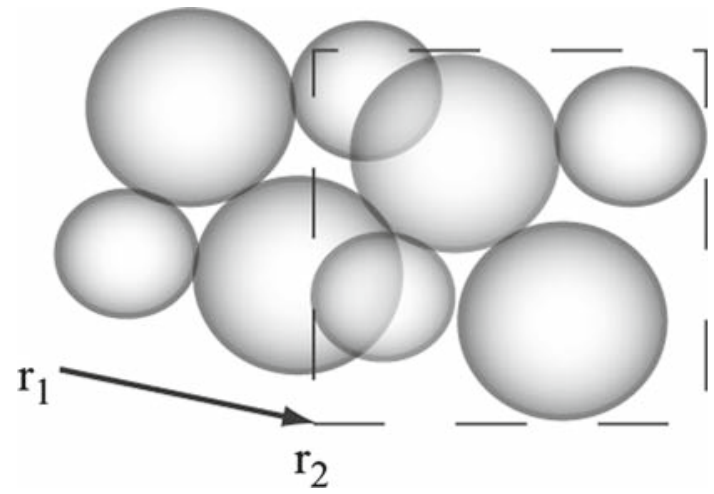

Fig. $1 \gamma(r)$ is the autocorrelation function of the density distribution. The correlation function $\gamma(r)$ depends on the shape/geometry and the structure of the sample. The function is a measure of the probability of being in the same phase after shifting from a point $r_{1}$ to a point $r_{2}$ in the sample $\left(r=\left|r_{1}-r_{2}\right|\right)$. This is equivalent to calculate the mean shared volume between an "image" and its ghost after shifting the ghost image some distance, $r$. For simple systems like a sphere and others, the correlation function is known analytically, but normally this function is calculated by numerical integration

and

$\gamma(r)=2 \int_{0}^{\infty} \Delta \rho\left(r^{\prime}\right) \Delta \rho\left(r^{\prime}+r\right) d r^{\prime}$,

being defined so that $\gamma(0)=1 . G(z)$ is measured around the mean-square fluctuations of the sample inhomogeneities. Above the characteristic size of the sample inhomogeneities no more correlations are expected and therefore we have that $G(\infty)=0$. The amplitude of the saturation level is

$P(\infty)=e^{-t \lambda^{2} \phi(1-\phi) \Delta \rho_{0}^{2} \xi}$

and depends on the correlation length $\xi$, the volume fraction $\phi$ of the sample inhomogeneities, on the chemical composition of the sample via $\Delta \rho_{0}$ and on the neutron wavelength $\lambda=2 \AA$ as well as on the sample thickness $t$.

The correlation length of the sample inhomogeneities is:

$\xi=2 \int_{0}^{\infty} \gamma(r) d r$

For a dilute gas of hard spheres we calculate $\xi=3 a / 2$, with radius $a$. Note that the interaction between neutrons and the sample takes place on the SLD differences in the sample $\Delta \rho$, being in this study between air (treated as vacuum) and the silica spheres. Correlations are measured around the excess SLD along the beam and for systems with high packing fractions or ordered system $G(z)$ may even exhibit negative values due to the excluded volume at the vicinity of particle surfaces. 


\subsection{Sample and experiment}

In the experiment we used a fine spherical silica powder, kindly provided by Kobo Products Inc. The manufacturer reported a wide lognormal size distribution with a peak at 3-4 $\mu \mathrm{m}$ spanning across a decade. The density of the primary grain does not necessarily correspond to the density of the material making up that grain. We performed $\mathrm{N}_{2}$ porosimetry measurements that concluded a primary grain density $\rho_{g} \simeq 0.45 \mathrm{~g} / \mathrm{cm}^{3}$ to be compared to $2.2 \mathrm{~g} / \mathrm{cm}^{3}$ for the density of pure silica. This density is in good agreement with the porosity stated by the manufacturer $\left(0.5 \mathrm{~g} / \mathrm{cm}^{3}\right)$.

SESANS experiments were carried out on a hollow cylindrical sample container filled with powder of known mass. Two samples were prepared with the same thickness $(5 \mathrm{~mm})$ but holding different mass of material, thus giving different volume fractions, $\phi \simeq 0.33$ and $\phi \simeq 0.75$ for the loose and the dense case respectively. The packing fractions are quoted in terms of their grain packing fractions, being

$\phi=\frac{\rho_{s}}{\rho_{g}}$,

where $\rho_{s}$ is the density of the sample (i.e., mass powder per volume sample container).

A simple uniaxial compression was applied to prepare the sample. The straining of the powder was done by hand. The rate of straining was a few millimeters per second.

To produce the loose sample, $\phi \simeq 0.33$, we carefully rained the powder into a cylindrical mould and sealed it off with glass and aluminium windows (Aluminium and quartz are transparent for neutrons).

The second measurement was conducted on a compressed sample, where the container was filled with more material, and the packing fraction was increased by compressing the powder along the neutron beam direction, $\phi \simeq 0.75$. We note that such a high packing fraction is possible due to the polydispersity in grain sizes. We also recognise an uncertainty in the measured porosity and in the determined volume-fractions, estimated to $\pm 5 \%$.

The experiments were conducted in ambient conditions and the moist level in the powder was determined to be $13 \%$ by weight ( $6 \%$ by volume), determined by weighing before and after drying. A reasonable value, given the amount of surface available for moist-adsorption.

The microstructure of the loose packed sample will be mainly given by the attractive van der Waals and capillary forces, rather than be driven by gravitational body forces and hard core repulsion. We do not expect the loose packed sample to settle or undergo compaction during the measurement.

The samples were left for several hours in the neutron beam in order to improve statistics in the recorded spectra. Because of the long measurement time we monitored

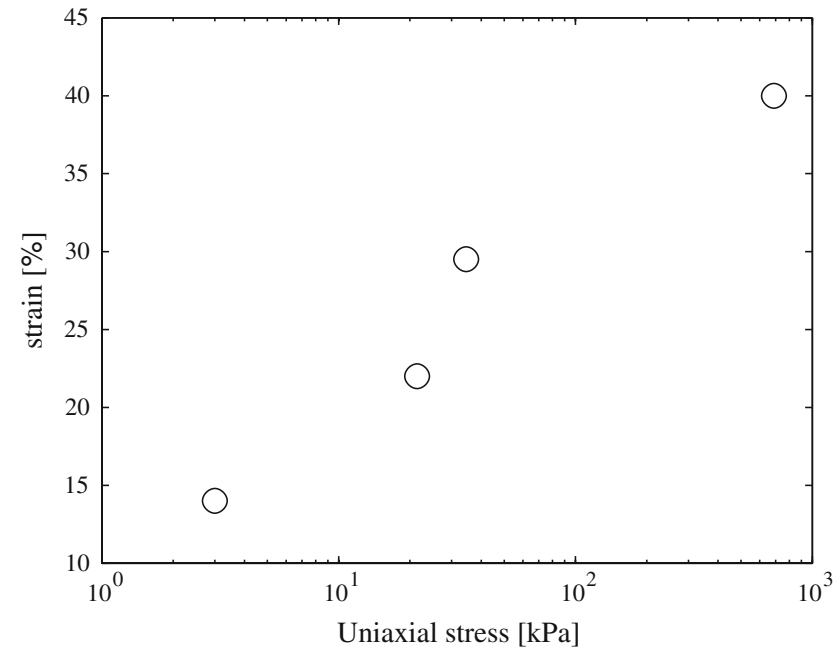

Fig. 2 Stress-strain curve obtained from uniaxial compression experiments on the silica powder

the stability (cracking, settling, compaction etc.) of the powder packing by visual inspection throughout the measurement. No compaction was observed on the loose packed sample during the measurement and the dense sample appeared homogeneous as far as one can see by eye. The pressure applied on the dense sample was about $100 \mathrm{kPa}$, determined by the constant weight acting on the plunger used to compress the powder.

A few points on the stress strain relationship for the powder was measured and is shown in Fig. 2. Plotting the volumetric strain $\left(\Delta V / V_{0}\right.$, change in volume over initial volume) as a function of the logarithm of the stress shows a linear relationship. It is a soft powder, easily strained, with an initially very low grain packing fraction.

\section{Molecular dynamics: method and model}

The interaction between grains in a granular material involves deformations at the contact point and in certain cases an attraction in the form of van der Waals attraction, capillary forces and electrostatic forces. Any computational attempt that convincingly models all possible interactions and deformations would be, to say the least, time consuming. Therefore we relate the interaction force to the overlap between particle pairs and particles interact only at contact (i.e., short-range forces). We used Molecular Dynamics simulations (MD) [21-23] to model the experiment. From the MD generated particle configuration we are able to calculate the corresponding SESANS correlation function $G(z)$ according to Eq. (2), and finally the expected polarisation with Eq. (1).

The MD code uses a linear hysteretic contact law, which allows for attractive/cohesive forces upon unloading [22,23], 


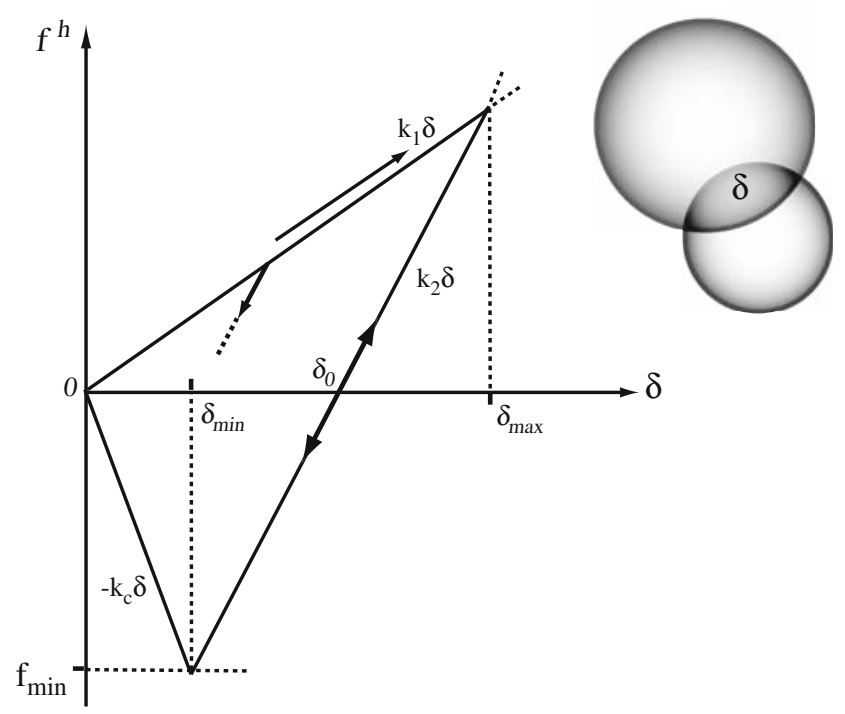

Fig. 3 Linear hysteretic force model. The left illustrates that at the contact point between two particles plastic deformations takes place in the form of permanent overlap. Unloading below $\delta_{0}$ gives rise to attraction between contacting pairs. The figure to the right shows two particles in contact producing an overlapping region

see Fig. 3. Three stiffness parameters govern the particleparticle contact model; $k_{1}, k_{2}$ and $k_{c}$ for loading, unloading and cohesive stiffness respectively. The initial loading path follows a linear increase $k_{1} \delta$ until a maximum overlap $\delta_{\max }$ is reached where $\delta_{\max }$ is a history parameter to be updated for each load cycle. For unloading, the force decreases along $k_{2} \delta$ until $\delta_{0}$

$\delta_{0}=\left(1-k_{1} / k_{2}\right) \delta_{\max }$.

Attractive/adhesive forces are activated when unloading below $\delta_{0}$. The force can be cast into one equation:

$f^{h}= \begin{cases}k_{1} \delta & \text { loading, if } k_{2}\left(\delta-\delta_{0}\right) \geq k_{1} \delta \\ k_{2}\left(\delta-\delta_{0}\right) & \text { un/reload, if } k_{1} \delta>k_{2}\left(\delta-\delta_{0}\right)>k_{c} \delta \\ -k_{c} \delta & \text { unloading, if }-k_{c} \delta \geq k_{2}\left(\delta-\delta_{0}\right)\end{cases}$

To allow for a stable integration of the equations of motion the timestep was chosen so that it resolves about 70 times the typical contact duration time, $t_{c}$, between a typical particle pair.

$t_{c}=\frac{\pi}{\omega}=\frac{\pi}{\sqrt{k_{2} / m_{12}-\eta^{2}}}$,

where $\omega$ is the eigen-frequency of the contact, $k_{2}$ the stiffness, $m_{12}=m_{1} m_{2} /\left(m_{1}+m_{2}\right)$ the reduced mass of the particle pair and $\eta=\gamma /\left(2 m_{12}\right)$ a damping coefficient. Energy is dissipated upon particle contacts and collisions, and also through the viscous damping coefficient $\gamma$, which (alone) leads to the coefficient of restitution, $r_{c}$ :

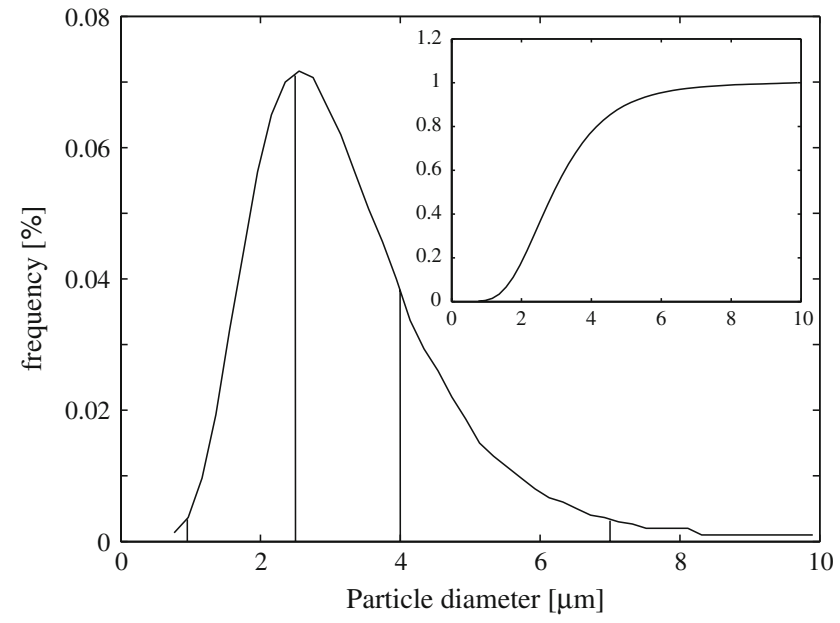

Fig. 4 The lognormal size distribution used in the simulation. The inset shows the cumulative probability distribution of sizes

$r_{c}=v^{\prime} / v=\exp \left(-\eta t_{c}\right)$

where $v$ and $v^{\prime}$ denote the particle velocity before and after a collision, respectively.

The particle size-distribution was chosen to be lognormal distributed with a mean at $3 \mu \mathrm{m}$ in terms of a sphere diameter $\left(R_{\max } / R_{\min }=15\right)$, see Fig. 4 . The particles were divided into five size-classes, indicated by the horizontal lines in Fig. 4, each holding a range of sizes of the lognormal distribution. The average coefficient of restitution for each size-class was set around $r_{c}=0.85$ by giving the particles different stiffness depending on their size.

Coulomb's law gives the tangential forces acting between particles, so that in the static case the tangential force is given by a static friction coefficient i.e., $f^{t} \leq \mu_{s} f^{n}$ and the sliding case is governed by a dynamic counter part $f^{d}=\mu_{d} f^{n}$. In general the relationship is $\mu_{d} \leq \mu_{s}$ and we use $\mu_{d}=\mu_{s}=0.4$.

Finally, an artificial background viscosity to dissipate the long-wavelength modes of motion is added; this damping can be attributed to a dispersion medium such as air, thus giving it a physical meaning rather than just enhancing the efficiency of the simulation [22].

The MD simulation carried out here contains two steps. First the initial preparation where we go from a packing fraction $\phi=0.08$ configuration to $\phi=0.33$ by increasing the radius of the particles. For an assembly of $N$ spheres with radius $a$ contained in volume $V$ we have $\phi=V^{-1} \sum_{i}^{N} 4 / 3 \pi a_{i}^{3}$. This preparatory step is followed by a uniaxial compression until we reach $\phi=0.75$. Table 1 summarizes the material properties and simulation characteristics at the start of wall movements. It is beyond the scope of this paper to discuss all parameters, details about the parameters can be found in [23]. 
Table 1 Material parameters and simulation parameters used in the MD simulation at the start of the wall movement. The stiffness and viscosity parameters are averages

\begin{tabular}{|c|c|c|c|}
\hline Parameter & Meaning & MD unit & SI unit (prefixed) \\
\hline$t$ & Time unit & 1 & $1 \mu \mathrm{s}$ \\
\hline$l$ & Length unit & 1 & $1 \mathrm{~m}$ \\
\hline$m$ & Mass unit & 1 & $1 \mathrm{~kg}$ \\
\hline$\langle D\rangle$ & Mean particle diameter & $3 \times 10^{-6}$ & $3 \mu \mathrm{m}$ \\
\hline$\delta$ & Particle density & 500 & $500 \mathrm{~kg} / \mathrm{m}^{2}$ \\
\hline$V$ & Total volume & $3 \times 10^{-12}$ & $3 \times 10^{-3} \mathrm{~mm}^{3}$ \\
\hline$\left\langle t_{c} / \Delta t\right\rangle^{\mathrm{b}}$ & Particle contact response time & 70 & - \\
\hline$\left\langle l_{\text {end }}\right\rangle /\langle D\rangle^{\mathrm{a}}$ & $\begin{array}{l}\text { Particle dist. travelled } \\
\text { over MD timestep (average) }\end{array}$ & 900 & $0.9 \mathrm{~mm}$ \\
\hline$\phi$ & Packing fraction & 0.33 & - \\
\hline$k_{2}$ & Elastic stiffness & $2 \times 10^{-7}$ & $2 \times 10^{5} \mathrm{~kg} / \mathrm{s}^{2}$ \\
\hline$k_{1}$ & Plastic stiffness & $0.2 k_{2}$ & \\
\hline$k_{c}$ & Cohesive stiffness & $10 k_{2}$ & \\
\hline$k_{t}$ & Friction stiffness & $0.2 k_{2}$ & \\
\hline$k_{r}$ & Rolling stiffness & $0.2 k_{2}$ & \\
\hline$k_{s}$ & Spinning stiffness & $0.2 k_{2}$ & \\
\hline$\mu_{d}=\mu_{s}$ & Coulumb friction coefficient & 0.4 & \\
\hline$\mu_{r}$ & Rolling friction & $\mu_{d}$ & \\
\hline$\gamma_{n}$ & Viscosity (normal) & $1 \times 10^{-12}$ & $1 \times 10^{-6} \mathrm{~kg} / \mathrm{s}$ \\
\hline$\gamma_{f}$ & Viscosity (tangential) & $0.25 \gamma_{n}$ & \\
\hline$\gamma_{r}$ & Viscosity (rolling) & $0.25 \gamma_{n}$ & \\
\hline$\gamma_{s}$ & Viscosity (spinning) & $0.25 \gamma_{n}$ & \\
\hline$\gamma_{b g t}$ & $\begin{array}{l}\text { Viscosity acting on } \\
\text { translations (background) }\end{array}$ & $0.1 \gamma_{n}$ & \\
\hline$\gamma_{b g r}$ & $\begin{array}{l}\text { Viscosity acting on } \\
\text { rotations (background ) }\end{array}$ & $0.05 \gamma_{n}$ & \\
\hline
\end{tabular}

a This is the average distance that the particles have travelled from the start of the simulation

b The timestep of the integration is chosen so that it can resolve the duration of particle contact
The longer ranged correlations are credited to the presence of clusters and voids with an average size above four particle diameters. Upon compacting, one expects the clusters to break up and voids will collapse. As can be seen in the top figure in Fig. 5 we reach a saturation in the correlations $\simeq 3 \mu \mathrm{m}$ for the dense sample with $\phi=0.75$, we attribute this size to the diameter of the particles, reported to be centred at $3 \mu \mathrm{m}$. The applied stress does not only break clusters and collapse voids, but it also give rise to excluded volume correlation among the grains as seen in the small dip around 2-3 $\mu \mathrm{m}$.

The amplitude of the saturation is proportional to the correlation length $\xi$ and the packing fraction $\phi$ of inhomogeneities in the sample according to Eqs. (4) and (5). Since the loose-packed sample never shows any saturation on the measured scale, it is hard to draw any quantitative conclusions; however, we note that the saturation level is below that of the compact sample leading to a higher correlation length, which is to be expected for a sample with larger voids. 

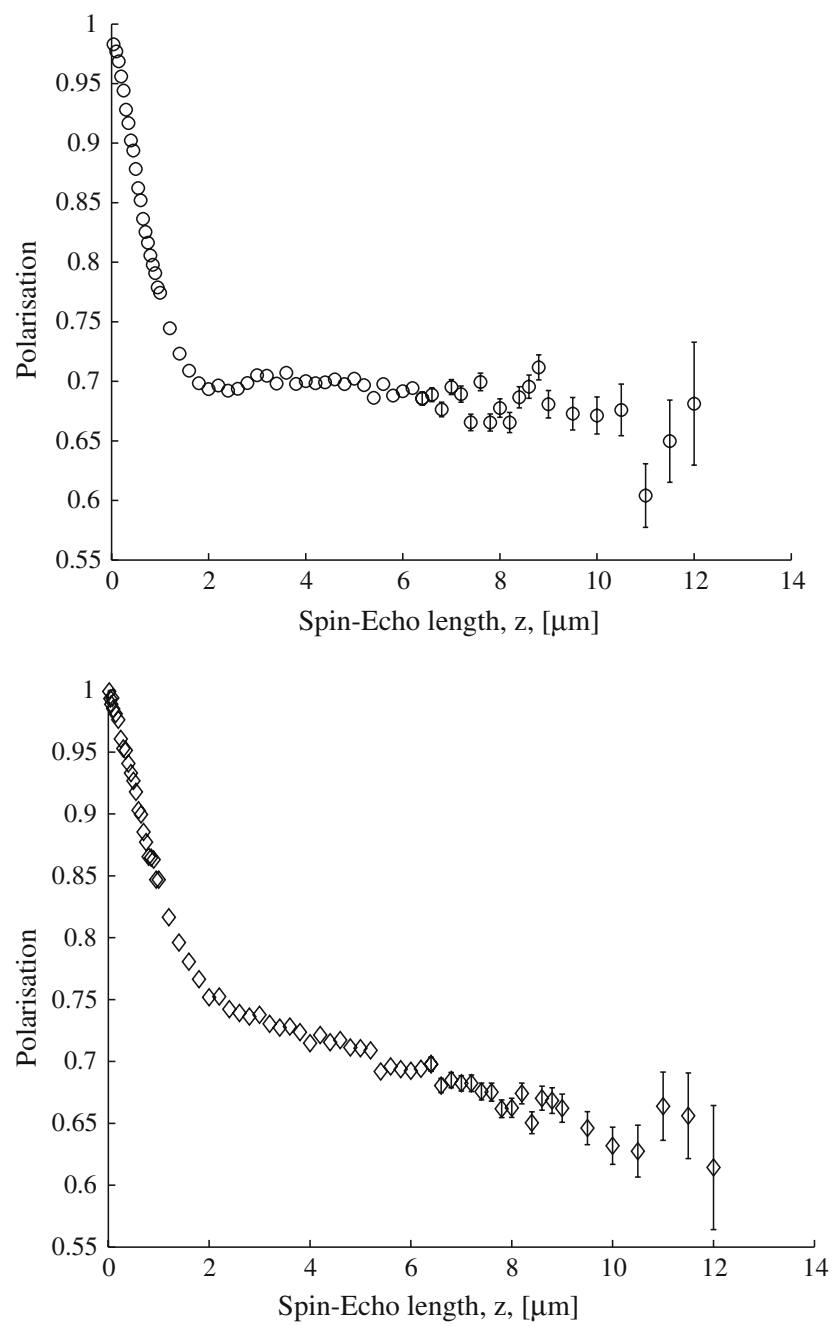

Fig. 5 Measurements on fine powders of silica, polarisation, $P(z)$, as function of spin-echo length $z$. The topmost plot is results obtained from the dense-packed sample and the lower figure represents the loosepacked sample. If not shown, all errors fall within markers

\subsection{Results: molecular dynamics}

We have used the MD method described in a previous section to carry out a uniaxial compression test on a low-volume fraction $(33 \%)$ cohesive powder packing. Simulations were carried out using a periodic cuboid undergoing a cosinusoidal (half period) compression along one dimension in zero gravity. The initial state was a random granular gas of low volume fraction $(\phi=0.08)$; with a Gaussian velocity distribution and in total carrying 3,000 particles. The initial state was allowed to grow (without compromising the size distribution) until a specified volume fraction was reached at $\phi=0.33$, and the final radius was $1.5 \mu \mathrm{m}$. On average the particle has travelled 900 average particle diameters at the end of the growth process. At this stage the simulation should represent the loose-packed sample in our experiment, holding a low volume fraction in a more clustered or open structure.
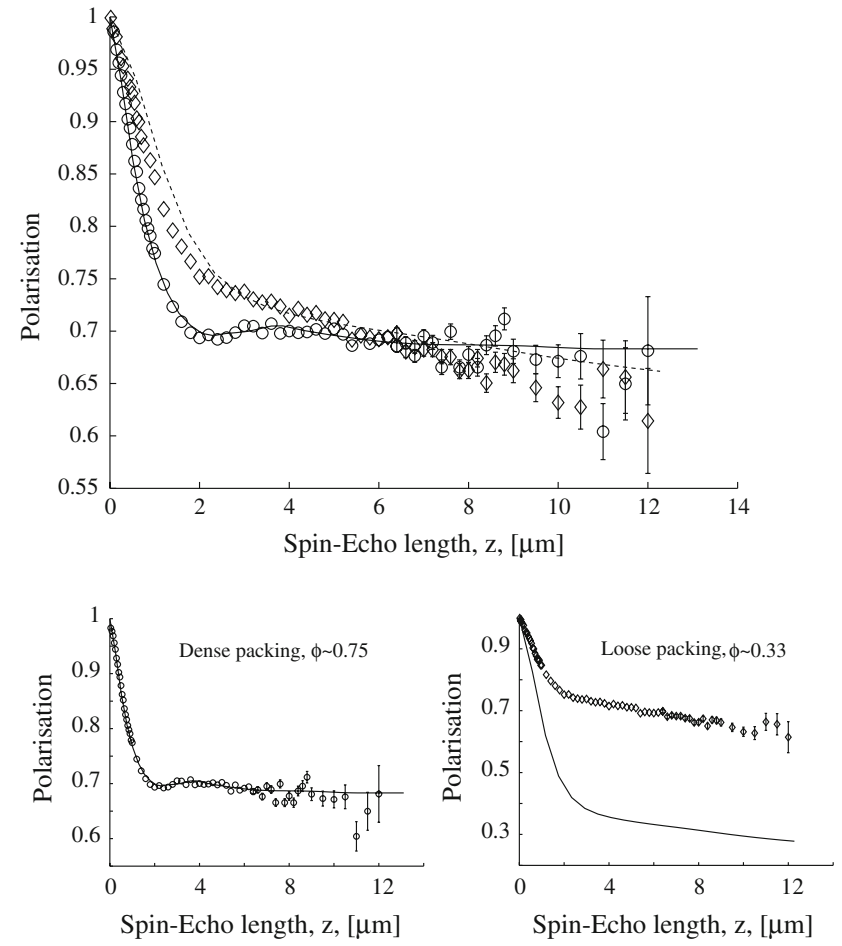

Fig. 6 Experimental and MD-simulation results. The top figure represents all measured and simulated results. Note that in the clustered case (diamonds) the simulation result (dashed line) is re-scaled to collapse on the measured points. We separate the two cases at the bottom and note only a qualitative agreement, on the shape, between the simulation and experiment for the loose-packed sample (rightmost figure). On the dense-packing we see a good quantitative agreement between measurement and simulations (leftmost figure). If not shown, all errors fall within the markers

We note that at the end of the simulation at $\phi=0.33$ the packing is not stable, but containing residual kinetic energy.

The loose-packed sample was compressed along one dimension to yield the final compressed state at $\phi=0.75$, for an illustration of the packings, see Fig. 7. In general, we conclude from Fig. 6 that the shapes of the curves are rather well reproduced when comparing the simulation with the experiments. The excluded volume feature seen in the experiment is also seen in the simulation in the dense case.

For the compressed state we calculate the correlation length to $\xi=1 \mu \mathrm{m}$, representing the typical size of inhomogeneities projected along the neutron beam, this is in excellent agreement with our measurement when taking into account the measured volume fraction, known SLD, the neutron wavelength and the sample thickness. The correlation function saturates on the horizontal axis around $z=3 \mu \mathrm{m}$ and this size represents the typical diameter of the spherical grains. All together, giving good agreement in both shape and amplitude of the polarisation in the dense case.

When comparing the uncompressed and compressed state in terms of $G(z)$ we note that the dense packing show a faster decay in the correlations thus, representing a sample 

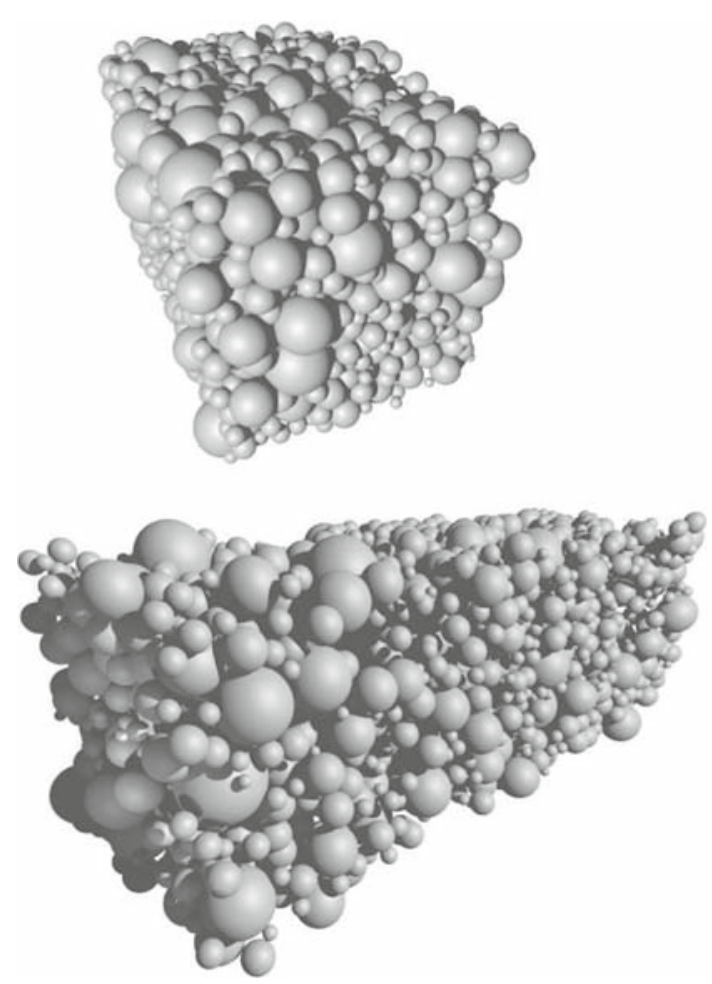

Fig. 7 Visualisation of: Top, the final compressed packing $(\phi=0.75)$ and bottom, the initial loosely packed state $(\phi=0.33)$

with shorter range in correlations, in essence a more closed system with smaller inhomogeneities.

For the simulation representing a loose-packed structure the calculated polarisation does not reproduce our experiment; however, we clearly see a correlation function with a good qualitative agreement in the overall shape (see the lower-left figure in Fig. 6). Note also the slope seen for higher $z$ in the experiment and seen in simulation on the loosepacked sample. The amplitude of the polarisation is given by the total amount of scattering according to Eq. (4) where mainly $\xi$ contributes to the amplitude (the other terms are constants, and $\phi(1-\phi)$ in this case, gives only a minor contribution. We extract from our simulation a correlation length, $\xi$, of 2.5 and $1 \mu \mathrm{m}$ for the loose and dense packing respectively. For a hard sphere gas we have $\xi=1.5$ times the sphere radius. We expect this correlation length to drop with increasing packing fraction.

We explain the discrepancy between measured and simulated polarisation on the loose packing by arguing that: in the experiment, we have a two-phase system of low/empty and -high density phases of particles so that the high-dense regions will contribute to a low-scattering amplitude via low $\xi$ and the low packing fraction is given by the large voids. At low length scales, $0.1 \mu \mathrm{m} \leq z \leq 3 \mu \mathrm{m}$, when comparing the experiment with our simulation result, we see in Fig. 6 a steeper slope in the correlation function (i.e., a more closepacked structure) and the argument here is that dense regions are given this behaviour. When we further compress the system we expect, at some stage, all clusters to break and larger voids to collapse, all together giving a final random densepacking of polydisperse spheres.

\section{Conclusions}

The use of spin-echo SANS for the study of structure in fine powders captures the structural differences in terms of the correlation function between loose and dense packings. The SESANS correlation function can be calculated from molecular dynamics simulations and compared with experiments. For the dense packing, we obtain a good quantitative agreement between measurements and simulations, whereas a qualitative agreement is obtained for the loose-packed system. In the loose packing we see no saturation of the correlations at larger length scales both in simulation and in experiment.

The SESANS correlation function is directly related to the density-density distribution function of the sample, through the projection along the neutron beam path. SESANS captures the transition between a loosely packed powder and a dense packing via a hard-core repulsion (excluded volume) feature in the correlation as well as in the correlation length and the overall curve-shape. The average size of the inhomogeneities can be extracted from the correlation function in the form of correlation length, $\xi$. The experiments show that the dense packing carries lower correlation length when compared to the loosely packed sample. This is expected, since the loose packed sample would mean a higher degree of clustering, giving rise to inhomogeneities and voids at larger length scale (this can also be concluded by visual inspection and by the fact that no saturation is observed). Interestingly, the correlations do not extend beyond the size of a grain in the dense packing. One expects the dense case to carry so called force networks, these networks (if they are present in the packing) do not create correlations in the density distribution.

The lognormal size distribution, used in the simulation, leads to the loss of excluded volume effects and nearestneighbour peaks in the correlation function, thus contributing to the agreement between measurement and simulation.

We acknowledge that the structure of the loose packed sample was not reproduced in our simulation. A stronger clustering mechanism than used in the simulation is needed, possibly by introducing an attractive potential. The simulation predicts a higher correlation length for the uncompressed state, which is expected for a more open and porous system. The total amount of neutron scattering and the correlation length is over-estimated when compared to our experiment. We argue that in the loose packed experiment we have a twophase system with large voids and large dense-clusters of particles (sub-millimeter sized). The dense clusters of particles would, in this case, contribute to a low correlation length and 
the voids give rise to the low packing fraction seen in the experiment.

We believe to have made a valuable connection between simulations and experiments, showing that in dense sphere packings with wide size distributions, the proposed MD model is in excellent agreement with the experiment. For loose cohesive powders, we need new models and larger simulations if we want capture the structure seen in real experiments.

Acknowledgments We thank Chris Duif at the Reactor Institute Delft for valuable discussions and help with performing the experimental part of this work. The authors would like to thank Sander Brouwer at DelftChemTech for performing the $\mathrm{N}_{2}$ porosimetry measurement on our silica powder. This work is part of the research programme of the 'Stichting voor Fundamenteel Onderzoek der Materie (FOM)', which is financially supported by the 'Nederlandse Organisatie voor Wetenschappelijk Onderzoek (NWO)'.

Open Access This article is distributed under the terms of the Creative Commons Attribution Noncommercial License which permits any noncommercial use, distribution, and reproduction in any medium, provided the original author(s) and source are credited.

\section{References}

1. Bernal, J.D., Mason, J.: Packing of spheres: co-ordination of randomly packed spheres. Nature 188, 910-911 (1960)

2. Visser, J.: Van der waals and other cohesive forces affecting powder fluidization. Powder Technol. 58(1), 1-10 (1989)

3. Luding, S.: Structure and cluster formation in granular media. Pramana J. Phys. 64(6), 893-902 (2005)

4. Tomas, J.: Fundamentals of cohesive powder consolidation and flow. Granular Matter 6, 75-86 (2004)

5. Sederman, A.J., Alexander, P., Gladden, L.F.: Structure of packed beds probed by magnetic resonance imaging. Powder Technol. 117, 255-269 (2001)

6. Mueth, D.M., Debregeas, G.F., Karczmar, G.S., Eng, P.J., Nagel, S.R., Jaeger, H.M.: Signatures of granular microstructure in dense shear flows. Nature 406, 385-389 (2000)
7. Toiya, M., Stambaugh, J., Losert, W.: Transient and oscillatory granular shear flow. Phys. Rev. Lett. 93(8), 088001 (2004)

8. Kohonen, M.M., Geromichalos, D., Scheel, M., Schier, C., Herminghaus, S.: On capillary bridges in wet granular materials. Phys. A 339(1-2), 7-15 (2004)

9. Aste, T., Saadatfar, M., Senden, T.J.: Geometrical structure of disordered sphere packings. Phys. Rev. E 71, 061302 (2005)

10. Richard, P., Philippe, P., Barbe, F., Bourlès, S., Thibault, X., Bideau, D.: Analysis by X-ray microtomography of a granular packing undergoing compaction. Phys. Rev. E 68, 020301R (2003)

11. Seidler, G.T., Martinez, G., Seeley, L.H., Kim, K.H., Behne, E.A., Zaranek, S., Chapman, B.D., Heald, S.M., Brewe, D.L.: Granuleby-granule reconstruction of a sandpile from X-ray microtomography data. Phys. Rev. E 62, 8175-8181 (2000)

12. Feigin, L.A., Svergun, D.I.: Structure analysis by small-angle X-Ray and neutron scattering. Plenum Press (1987)

13. Rekveldt, M.T.: Novel SANS instrument using neutron spin echo. Nucl. Instr. Meth. Phys. Res. B 114, 366-370 (1996)

14. Rekveldt, M.T.: Neutron reflectometry and sans by neutron spin echo. Phys. B 234, 1135-1137 (1997)

15. Rekveldt, M.T., Plomp, J., Bouwman, W.G., Kraan, W.H., Grigoriev, S., Blaauw, M.: Larmor precession applications: magnetised foils as spin flippers in spin-echo sans with varying wavelength. Rev. Sci. Ins. 76, 033901 (2005)

16. Andersson, R., Bouwman, W.G., de Schepper, I.M.: Superfine powders of silica studied with spin-echo small-angle neutron scattering. In: Powders and Grains 2005, pp. 13-15. Stuttgart, Germany (2005)

17. Andersson, R., Bouwman, W.G., Luding, S., de Schepper, I.M.: Stress, strain and bulk microstructure in a cohesive powder. Phys. Rev. E 77, 051303 (2008)

18. Gähler, R., Golub, R., Habicht, K., Keller, T., Felber, J.: Spacetime description of neutron spin echo spectrometry. Physica B 229, 1-17 (1996)

19. Tromp, H., Bouwman, W.G.: A novel application of neutron scattering on dairy products. Food Hydrocolloids 21, 154-158 (2007)

20. Tromp, H., Bouwman, W.G.: Neutron News. 3(3), 29-37 (1992)

21. Pöschel, T., Schwager, T.: Comput. Granular Dynam. Springer, Berlin (2005)

22. Luding, S.: Anisotropy in cohesive, frictional granular media. J. Phys. Condensed Matter 17, 2623-2640 (2005)

23. Luding S. (2005) Cohesive, frictional powders: contact models for tension. Granular Matter 10(4), 235-246 (2008) 